\title{
Biological Performance of a Bioabsorbable Magnesium-magnesium Phosphate Cement Interbody Fusion Cage in a Porcine Lumbar Interbody Fusion Model: A Feasibility Study
}

\section{Xuxuan Wang}

Chinese PLA General Hospital

Yabin Zhang

Chinese PLA General Hospital

Yihao Liu

Chinese PLA General Hospital

\section{Xiucan Li}

Chinese PLA General Hospital

Keya Mao ( $\nabla$ maokeya@sina.com )

Department of Orthopedics, First Medical Center ofChinese PLA General Hospital, 28 Fuxing Road, Beijing

\section{Research article}

Keywords: Bioabsorbable cage, Magnesium, Magnesium phosphate cement, Porcine animal model, Degradation

Posted Date: July 22nd, 2020

DOI: https://doi.org/10.21203/rs.3.rs-41956/v1

License: (c) (i) This work is licensed under a Creative Commons Attribution 4.0 International License. Read Full License 


\section{Abstract \\ Background}

Magnesium phosphate cement (MPC) is a novel biodegradable bone adhesive. The major drawback of the Magnesium phosphate cement (MPC) cage for implantation is its high brittleness. The magnesium was presumed to compensate for the lack of toughness in the MPC cage. The aim of the study was to evaluate the feasibility of a bioabsorbable cage consisting of magnesium and magnesium phosphate cement (MPC) in a porcine lumbar interbody fusion model.

\section{Methods}

Twelve male Ba-Ma mini pigs underwent lumbar discectomy and fusion with an Mg-MPC cage or a PEEK cage at the L3/L4 and L4/L5 level. Computed tomography (CT) scans were made to evaluate the distractive property by comparing average disc space height (DSH) before and at 6, 12, and 24 weeks after the operation. After the lumbar spines were harvested at 6 or 24 weeks after the operation, micro-CT examination was conducted to analyze the fusion rate, and stiffness of motion segments was investigated through mechanical tests. A histological study was performed to evaluate the tissue type in the intervertebral space.

\section{Results}

CT scans showed no significant difference between the two groups in average DSH at each time point. Micro-CT scans revealed an equal fusion rate in both groups ( $0 \%$ at 6 weeks, $83.3 \%$ at 24 weeks). Both groups showed time-dependent increases in stability, the Mg-MPC cages achieved an inferior stiffness at 6 weeks and a comparable stiffness at 24 weeks. Histologic evaluation showed the presence of newly formed bone in both groups. However, empty spaces were observed at the interface or around the Mg-MPC cages.

\section{Conclusion}

Compared with the PEEK cages, the Mg-MPC cages achieved comparable distraction, fusion rate, and spinal stability at 24 weeks after the operation. However, due to inferior stiffness at the early stage and fast degradation, further modification of material composition and design are necessary.

\section{Background}

Magnesium phosphate cement (MPC) is a novel biodegradable bone adhesive that could be used to directly conglutinate small fragments of non-loaded bone [1, 2]. However, no research on the use of MPC as an interbody fusion cage is available. Our previous research on MPC cages in a porcine lumbar spine 
interbody fusion model showed intervertebral fusion and conspicuous degradation of MPC at 6 months after implantation. The major drawback of the MPC cage for implantation is its high brittleness. In order to implant an MPC cage in the intervertebral space without cracking, the intervertebral space needs to be made broad enough to accommodate the cage, which is likely to cause damage to the vertebral endplate.

Magnesium is attractive for its degradation ability, elastic modulus similar to that of bone, stimulatory effect on bone growth, and good biocompatibility. Moreover, the fracture toughness of magnesium is greater than that of ceramic biomaterials such as hydroxyapatite [3].

The idea to combine MPC and magnesium in a hybrid cage (Mg-MPC cage) was conceived to ensure adequate initial toughness for operation and gradual degradation and osteoconduction for bone replacement.

This feasibility study was aimed at observing the fusion and degradation performance of the Mg-MPC cage, evaluating the histological and biomechanical properties in a porcine lumbar spine interbody fusion model.

\section{Materials And Methods}

\section{Materials}

The MPC powder used in this study was composed of magnesium oxide ( $\mathrm{MgO})$, ammonium dihydrogen phosphate $\left(\mathrm{NH}_{4} \mathrm{H}_{2} \mathrm{PO}_{4}\right)$, and sodium borate $\left(\mathrm{Na}_{2} \mathrm{~B}_{4} \mathrm{O}_{7}\right)$. The $\mathrm{MgO}$ and the $\mathrm{NH}_{4} \mathrm{H}_{2} \mathrm{PO}_{4}$ were in a molar ratio of 3.8:1. The $\mathrm{MgO}$ was prepared by heating basic magnesium carbonate pentahydrate $\left[4 \mathrm{MgCO}_{3} \cdot \mathrm{Mg}(\mathrm{OH})_{2} \cdot 5 \mathrm{H}_{2} \mathrm{O}\right.$ ] in a furnace at $1500^{\circ} \mathrm{C}$ for $6 \mathrm{~h}$. After cooling and sieving process, the grains in the range of 200 and 300 meshes were kept for further experiment. Deionized water was employed as the cement liquid. The powder-to-liquid (P/L) ratio was $6.25 \mathrm{~g} / \mathrm{ml}$. The MPC powder was mixed with deionized water for 2 minutes to form paste, which was injected into a stainless-steel mold internally covered with magnesium bracket using a pressing device. The magnesium bracket was made of pure magnesium in the integrated molding process, and the purity of magnesium was more than $99 \%$. The magnesium bracket was $20 \mathrm{~mm}$ long, $8 \mathrm{~mm}$ wide, $6 \mathrm{~mm}$ high, and $0.5 \mathrm{~mm}$ thick. After setting for $30 \mathrm{~min}$, the Mg-MPC cages were dried at $60^{\circ} \mathrm{C}$ for $24 \mathrm{~h}$ and sealed with a PE bag. The Mg-MPC cage was a block cage, which was $20 \mathrm{~mm}$ long, $8 \mathrm{~mm}$ wide, and $6 \mathrm{~mm}$ high (Fig. 1a). All the chemicals used for the Mg-MPC cages were purchased from Ningbo Hicren Biotechnology co., Ltd, China. The PEEK cage was a box cage, manufactured by Johnson \& Johnson (Johnson \& Johnson Medical Devices Companies, USA), which was $15 \mathrm{~mm}$ long, $12 \mathrm{~mm}$ wide, and $6 \mathrm{~mm}$ high, with a 3.5- $\mathrm{mm}$ radius central hole (Fig. 1b). The plants were sterilized by low-temperature plasma sterilization prior to the operation.

Animals and experimental design

Twelve male Ba-Ma mini pigs (weight 25-30 kg; average age 6 months) were used for this study with study time points of 6,12 , and 24 weeks after the operation. Each animal underwent extreme lateral 
interbody fusion (XLIF) with an Mg-MPC cage or a polyetheretherketone (PEEK) cage for control at the levels of $L 3 / L 4$ and L4/L5. The two kinds of cages were inserted at the L3/L4 or L4/L5 level randomly. $A$ $1-\mathrm{cm}$ transverse process was cut off from the tip of $L 4$ for the autologous bone grafts. PEEK cages were implanted with the central holes filled with autologous transverse process bone grafts. After implantation, titanium plates and screws were applied for fixation. The animals were sacrificed at 6 weeks $(n=6)$ and 24 weeks $(n=6)$ after the procedure. The spine specimens of interest were harvested and stored at $20{ }^{\circ} \mathrm{C}$ for further tests.

\section{Computed tomography}

CT was performed using a CT scanner (GE, Optima CT 660, USA) before and at 6, 12, and 24 weeks after surgery. Axial cuts with 1-mm slice thickness were made parallel to the intervertebral disc space. At those time points, the anterior, middle, and posterior intervertebral disc space height (DSH) was measured from the middle sagittal view. The average DSH was calculated from anterior, middle, and posterior DSH measurements by the following formula: average DSH = (anterior DSH + middle DSH + posterior DSH)/3 (Fig. 2) [4].

\section{Micro-computed tomography}

The surgically treated motion segments were dissected and labeled. The microstructure of specimens was observed with micro-CT (PerkinElmer-Caliper LS, Quantum FX Demo, USA). The fusion rate was analyzed by observing the bony bridging of the discectomy space from the sagittal view of the specimen.

\section{Mechanical tests}

Mechanical properties were tested as described previously by Panjabi et al. [5]. The specimens at 6 weeks $(n=6)$ and 24 weeks $(n=6)$ after the operation underwent mechanical tests. Three fresh mini pig cadaveric lumbar spines (6 motion segments) were used as control. The specimens were thawed in a $4{ }^{\circ} \mathrm{C}$ refrigerator and covered with a polyethylene warp to prevent dehydration. Each motion segment to be tested consisted of a cranial and a caudal lumbar vertebra. The fixation devices were removed. The facet joints and posterior elements of the motion segment were removed prior to testing. The cranial and caudal vertebra were fixed on a universal testing machine (Instron, 55MT, USA). Specimens were tested for flexion/extension, right/left lateral bending, and right/left axial rotation sequentially at a constant loading rate (1.0 degree/s) and a maximum torque of $5 \mathrm{~N} \mathrm{~m}$. The stiffness was calculated as moment $(\mathrm{Nm})$ /rotation angle (degree). Five consecutive readings were recorded and the last record was used for statistical analysis.

Histology

After the mechanical tests, the specimens were dehydrated in graded ethanol and embedded in plastic films. The specimens were cut into longitudinal sections by precision saw (Leica, SP1600, Germany) and ground into parallel sections of $40-\mu \mathrm{m}$ thickness. Tissue sections were stained with Giemsa staining and Masson trichrome staining and then observed by light microscope. The intervertebral space was 
analyzed using a slightly modified protocol from Zdeblick et al. [6]. Briefly, the tissue type at the implantvertebra interface or around the implant was rated as empty/fibrous tissue/bone [7, 8].

Statistical methods

Measurement data are expressed as mean \pm standard deviation. Enumeration data are expressed as number of cases. Statistical analysis was performed using SPSS 19.0 for windows (SPSS Inc., USA). The Student's $t$-test, one-way ANOVA, Scheffe's test, and chi-square test were used to compare the values among groups. $P<0.05$ was considered as statistically significant.

\section{Results}

\section{Computed tomography}

The preoperative average DSH did not show a significant difference between the two groups $(P>0.05)$. Moreover, there were no significant differences in average DSH between the two groups at 6, 12, and 24 weeks after the operation ( $P>0.05$; Figs. 3 and 4 ). No gas accumulation was observed at 6,12 , and 24 weeks after the operation.

\section{Micro-computed tomography}

At 6 weeks, both the Mg-MPC cage and the PEEK cage attached to the endplate and newly formed bone tissue was observed to infiltrate into the interface. Cracks and cavities formed on the surface of the Mg-MPC cage, indicating degradation of the Mg-MPC. Both the two groups remained unbridged (Fig. 5).

At 24 weeks, scanning images from the sagittal view revealed the use of an Mg-MPC cage or a PEEK cage led to an equal spinal fusion rate: the fusion rate is $83.3 \%$ in both cases $(P>0.05$; Table 1$)$. Newly formed bone tissue traversed the Mg-MPC cage to bridge the discectomy space, with the Mg-MPC cage degrading simultaneously (Fig. 6). Due to fixing system failure, cage rotation and movement were observed in one specimen of the Mg-MPC group and of the PEEK group, respectively, which led to spinal fusion failure.

Table 1

\begin{tabular}{|l} 
Micro-CT evaluation of fusion rate \\
\begin{tabular}{|lll|}
\hline Time point & Mg-MPC & PEEK \\
\hline 6 weeks & $0 / 6$ & $0 / 6$ \\
\hline 24 weeks & $5 / 6$ & $5 / 6$ \\
\hline
\end{tabular}
\end{tabular}

Mechanical tests

At 6 weeks, both the Mg-MPC group and the PEEK group showed a higher stiffness in all loading directions (flexion and extension, right and left lateral bending, and right and left axial rotation) compared 
to the negative control (normal lumbar segments) $(P<0.05)$. Compared with the Mg-MPC group, the stiffness of the PEEK group in flexion and extension and in the lateral bending direction is higher $(P<$ 0.05), indicating that a better spinal stability was achieved in the PEEK group.

At 24 weeks, both the Mg-MPC group and the PEEK group showed a further increase in stiffness compared to the negative control $(P<0.05)$. Meanwhile, the Mg-MPC group and the PEEK group achieved comparable spinal stability performance in all loading directions $(P>0.05$; Figs. $7-9)$.

Histology

Large defects were observed in histological sections of the Mg-MPC cages, which were formed during sectioning due to brittleness.

In the Mg-MPC group, bone tissue started to protrude into the implant-vertebra interface at 6 weeks after the operation. At 24 weeks after the operation the bone growth progressed both in the interface and around the implant. Despite the new bone formation, empty spaces could be observed in the interface or around the Mg-MPC cages due to fast degradation of the implant.

In the PEEK group, at 6 weeks after the operation new bone seemed to be absent and the implants were firmly bonded to the endplates by fibrous tissue. At 24 weeks after the operation, new bone accompanied by fibrous tissue had formed both at the interface and around the implants. The fibrous tissue was observed in large number of tissue sections. (Figs. 10 and 11).

\section{Discussion}

The ideal bioabsorbable materials for cage devices should possess three properties: the capability of degradation during a distinct period, the property not to obscure postoperative radiological estimation of intervertebral fusion, and the stiffness comparable with that of bone. During the degradation process, loading is transferred gradually to the newly formed bone and the cage is replaced with bone. Several previous studies involved animal experiments or clinical trials with bioabsorbable cages [9-16]. Most of these bioabsorbable cages were composed of polylactic acid $[9-11,15]$, which naturally degrades to carbon dioxide and water. Other bioabsorbable substances are polymer-calcium phosphate composites, composites of polyglycolic acid, multiamino acid copolymer/nanohydroxyapatite/calcium sulfate, and multiamino acid copolymer/a-tricalcium phosphate [17-19]. Although most of these studies demonstrated successful fusion with these cages, some adverse events were reported. Kandziora et al. [4] showed that poly-L-lactide-co-D, L-lactide (PLDLLA) cages were associated with osteolysis in a sheep model at 12 weeks after the operation. They found a severe foreign body reaction causing osteolysis around the PLDLLA cage. The osteolysis caused skepticism about the value of PLDLLA as an implant.

MPC has attracted much attention as a potential biodegradable bone implant material. The main components of MPC are magnesium oxide (MgO) and acid ammonium phosphates, particularly ammonium dihydrogen phosphate $\left(\mathrm{NH}_{4} \mathrm{H}_{2} \mathrm{PO}_{4}\right)$. MPC powders react in the presence of water to form 
$\mathrm{HN}_{4} \mathrm{MgPO}_{4} \cdot 6 \mathrm{H}_{2} \mathrm{O}$ (struvite) as the final product [2]. Yu et al. [20] assessed the toxicology and biocompatibility of MPC through the UDS test, Ames test, and micronuclei test and also evaluated degradation and osteogenesis in a rabbit femur condyle defect model. The results showed that MPC did not induce DNA damage or gene mutations. As regards the implantation test, the MPC implant formed direct bonds with the host bone and exhibited good degradation at 6 months after surgery. Our previous studies on a pure MPC cage also showed intervertebral fusion and conspicuous degradation of MPC at 6 months after implantation in a porcine lumbar spine interbody fusion model. However, the major drawback of the pure MPC cage as an implant is its high brittleness, prompting further modification to increase the toughness.

Magnesium is the fourth most abundant cation in the human body, with approximately half of the total physiological magnesium stored in bone tissue [3]. The elastic modulus and compressive yield strength of magnesium are closer to those of natural bone, while the fracture toughness of magnesium is greater than that of ceramic biomaterials such as hydroxyapatite [3]. In addition, magnesium exerts stimulatory effects on the growth of new bone tissue [21,22]. The major drawback of magnesium as an implant is the quick corrosion in the high chloride environment at physiological $\mathrm{pH}$ (7.4-7.6), losing mechanical integrity before the tissue has sufficiently healed. Moreover, magnesium produces hydrogen gas in the corrosion process that is not likely to be dealt with by the host tissue immediately [21]. Daentzer et al. [23]

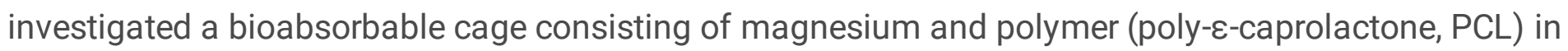
an ovine cervical model. The postoperative lateral radiographs showed gas accumulation in front of the disc space in $50 \%$ of the sheep. The gas completely disappeared within the next 3 weeks. In this study, no gas accumulation was observed at 6,12 , and 24 weeks after the operation, because the amount of magnesium in the hybrid cage was small, so hydrogen gas could easily be dealt with by the host tissue.

To our knowledge, this study is the first attempt to use MPC and magnesium as an interbody fusion cage to assist intervertebral fusion in vivo. The magnesium was employed to compensate for the lack of toughness in the MPC cage. Compared to a pure MPC cage, the hybrid Mg-MPC cage could be easily placed into the intervertebral space without fragmentation during the operation.

Cages should retain interbody distraction and resist against subsidence into the adjacent vertebra to guarantee bony fusion of a desired quality. The distractive properties of cages have been investigated in some experiments. Kandziora et al. [4] showed that a PLDLLA cage and autologous tricortical iliac crest bone graft developed a remarkable loss of DSH during an observation period of 12 weeks in a sheep cervical spine fusion model due to degradation of the implant and gradual graft collapse, respectively. Jiya et al. [16] assessed clinical and radiologic outcomes of the PLDLLA cage compared with the PEEK cage, demonstrating the PEEK cage group had a lower subsidence rate. Our study demonstrated both PEEK cages and Mg-MPC cages were able to distract intervertebral disc spaces beyond their baseline measure at 6 weeks after the operation. However, the average DSH decreased significantly in both groups at 12 and 24 weeks after the operation. Too rigorous preparation of the endplates might leave a mechanically weaker spongious bone bed to cages, thus promoting subsidence in both groups. Moreover, the decrease in DSH in the Mg-MPC group resulted partly from degradation. 
Assessment of fusion was frequently based on radiographs or histology. Toth et al. [24] introduced a fusion scoring system by using high resolution radiographs. Zdeblick et al. [6] devised a grading scale to analyze the histologic fusion results. The two methods have been widely applied to analyze fusion rates in animal experiments $[4,19]$. Micro-CT is more conducive to estimate the trabecular structure of newly formed bone. In this study, at 24 weeks in spite of the assistance of autologous transverse process bone grafts in the PEEK group, the Micro-CT scans revealed an equal fusion rate in both groups. Moreover, the newly formed bone tissue at the implant-vertebra interface in the Mg-MPC group seemly denser than that in the PEEK group probably due to the stimulatory effect of $\mathrm{Mg}$ on bone growth.

Compared to Mg-MPC group, the PEEK group showed higher stiffness in the flexion, extension, and lateral bending directions at 6 weeks after the operation. In addition to the large amount of fibrous tissue that is likely to increase the spinal stiffness, the autograft bone in the central hole of the PEEK cages may lead to quicker bone growth, improving the stability at the early stages. Other studies also revealed using bone graft was likely to affect the interbody fusion [25]. Li et al. [19] observed that titanium cages packed with autologous iliac crest bone grafts in the central hole could achieve quicker fusion than polycaprolactone-tricalcium phosphate (PCL-TCP) cages without a central hole. With the knowledge gained from these results, further research is recommended to investigate if adding a central hole in the Mg-MPC cage, which could be filled with bone grafts, can help achieve quicker fusion as well as better stability.

The histological study revealed that both the Mg-MPC cage and the PEEK cage did not behave well in terms of osseointegration, that is, empty spaces were observed at the implant-vertebra interface or around the implant due to relatively rapid degradation of the Mg-MPC cage, while the PEEK cages were surrounded by large amounts of fibrous tissue which may be a result of a foreign body reaction. The rapid degradation seems to be another possible cause of the insufficient stability at the early stage in the $\mathrm{Mg}-\mathrm{MPC}$ group. The degradation rate of the bioabsorbable implants is related to the material composition, the structure, and the degradation environment [26]. Therefore, the degradation of the Mg-MPC cage could be improved by modification of the material composition or the structure of the implant.

The limitations of this study include the limited numbers of animals enrolled in each group and the time points chosen for evaluation. The follow-up period does not allow for a description of the performance of $\mathrm{Mg}-\mathrm{MPC}$ cages in the long term. In addition, due to different designs of the two kinds of cages, whether the bone grafts were used, and the degradation of the Mg-MPC cages, we couldn't take an appropriate approach to make a quantitative analysis of the tissue type, particularly the newly formed bone tissue between the two groups. Moreover, in order to create enough space to accommodate the cages, the endplates were prepared too large in the two groups, which could induce interference in the evaluation of the distractive properties of the two kinds of cages.

\section{Conclusion}


In this feasibility study, we demonstrated that in contrast to PEEK cages, the Mg-MPC cages achieved comparable distraction, fusion rate, and spinal stability at 24 weeks after the operation. However, due to the inferior stiffness at the early stage and fast degradation, further modification of material composition and design are necessary. Moreover, the drawbacks mentioned above in this study should be improved.

\section{Abbreviations}

MPC

Magnesium phosphate cement

$\mathrm{Mg}$

Magnesium

PEEK

Polyetheretherketone

DSH

Average disc space height

CT

Computed tomography

PLDLLA

poly-L-lactide-co-D, L-lactide

\section{Declarations}

\section{Ethics approval and consent to participate}

All animal procedures were performed under the approval and guidance of the Animal Ethnics Committee of Chinese PLA General Hospital (approval number: E170406).

\section{Consent for publication}

Not applicable

\section{Availability of data and materials}

The datasets used and/or analysed during the current study are available from the corresponding author on reasonable request.

\section{Competing interests}

The authors declare that they have no competing interests.

\section{Funding}


This work was supported by the National Natural Science Foundation of China (No. 51372276, No. 51772328).

\section{Authors' contributions}

$\mathrm{XW}$ participated in the design of the study, performed the experiments, and wrote the manuscript. $\mathrm{YZ}, \mathrm{XL}$, and $Y L$ assisted to perform the experiments. $X W$ and $X L$ participated in the data monitoring, statistical analysis, and figure making. KM conceived the study, participated in its design and coordination. The authors read and approved the final manuscript.

\section{Acknowledgements}

The authors would like to acknowledge Dr. Xuepin Li for experimental support.

\section{Authors' information}

\section{Affiliations}

Department of Orthopedics,First Medical Center of Chinese PLA General Hospital, Beijing, People's Republic of China

Xuxuan Wang, Yabin Zhang, Yihao Liu, Xiucan Li, and Keya Mao

\section{References}

1. Waselau M, Samii VF, Weisbrode SE, Litsky AS, Bertone AL. Effects of a magnesium adhesive cement on bone stability and healing following a metatarsal osteotomy in horses. Am J Vet Res. 2007;68(4):370-8.

2. Wu F, Wei J, Guo H, Chen F, Hong H, Liu C. Self-setting bioactive calcium-magnesium phosphate cement with high strength and degradability for bone regeneration. Acta Biomater. 2008;4(6):187384.

3. Staiger MP, Pietak AM, Huadmai J, Dias G. Magnesium and its alloys as orthopedic biomaterials: a review. Biomaterials. 2006;27(9):1728-34.

4. Kandziora F, Pflugmacher R, Scholz M, Eindorf T, Schnake KJ, Haas NP. Bioabsorbable interbody cages in a sheep cervical spine fusion model. Spine (Phila Pa 1976). 2004;29(17):1845-55. discussion 56.

5. Panjabi MM. Biomechanical evaluation of spinal fixation devices: I. A conceptual framework. Spine (Phila Pa 1976). 1988;13(10):1129-34.

6. Zdeblick TA, Ghanayem AJ, Rapoff AJ, Swain C, Bassett T, Cooke ME, et al. Cervical interbody fusion cages. An animal model with and without bone morphogenetic protein. Spine (Phila Pa 1976). 1998;23(7):758-65. discussion 66. 
7. Anderson JM, Rodriguez A, Chang DT. Foreign body reaction to biomaterials. Semin Immunol. 2008;20(2):86-100.

8. Trindade R, Albrektsson T, Tengvall P, Wennerberg A. Foreign Body Reaction to Biomaterials: On Mechanisms for Buildup and Breakdown of Osseointegration. Clin Implant Dent Relat Res. 2016;18(1):192-203.

9. van Dijk M, Smit TH, Burger EH, Wuisman PI. Bioabsorbable poly-L-lactic acid cages for lumbar interbody fusion: three-year follow-up radiographic, histologic, and histomorphometric analysis in goats. Spine (Phila Pa 1976). 2002;27(23):2706-14.

10. Lanman TH, Hopkins TJ. Early findings in a pilot study of anterior cervical interbody fusion in which recombinant human bone morphogenetic protein-2 was used with poly(L-lactide-co-D,L-lactide) bioabsorbable implants. Neurosurg Focus. 2004;16(3):E6.

11. Frost A, Bagouri E, Brown M, Jasani V. Osteolysis following resorbable poly-L-lactide-co-D, L-lactide PLIF cage use: a review of cases. Eur Spine J. 2012;21(3):449-54.

12. Tunc DC, van Dijk M, Smit T, Higham P, Burger E, Wuisman P. Three-year follow-up of bioabsorbable PLLA cages for lumbar interbody fusion: in vitro and in vivo degradation. Adv Exp Med Biol. 2004;553:243-55.

13. van Dijk M, Tunc DC, Smit TH, Higham P, Burger EH, Wuisman PI. In vitro and in vivo degradation of bioabsorbable PLLA spinal fusion cages. J Biomed Mater Res. 2002;63(6):752-9.

14. Daentzer D, Willbold E, Kalla K, Bartsch I, Masalha W, Hallbaum M, et al. Bioabsorbable interbody magnesium-polymer cage: degradation kinetics, biomechanical stiffness, and histological findings from an ovine cervical spine fusion model. Spine (Phila Pa 1976). 2014;39(20):E1220-7.

15. Pflugmacher R, Eindorf T, Scholz M, Gumnior S, Krall C, Schleicher P, et al. [Biodegradable cage. Osteointegration in spondylodesis of the sheep cervical spine]. Chirurg. 2004;75(10):1003-12.

16. Jiya T, Smit T, Deddens J, Mullender M. Posterior lumbar interbody fusion using nonresorbable polyether-ether-ketone versus resorbable poly-L-lactide-co-D,L-lactide fusion devices: a prospective, randomized study to assess fusion and clinical outcome. Spine (Phila Pa 1976). 2009;34(3):233-7.

17. Chunguang Z, Yueming S, Chongqi T, Hong D, Fuxing P, Yonggang Y, et al. Evaluation of bioabsorbable multiamino acid copolymer/alpha-tri-calcium phosphate interbody fusion cages in a goat model. Spine (Phila Pa 1976). 2011;36(25):E1615-22.

18. Ren C, Song Y, Xue Y, Yang X, Zhou C. Evaluation of Bioabsorbable Multiamino Acid Copolymer/Nanohydroxyapatite/Calcium Sulfate Cage in a Goat Spine Model. World Neurosurg. 2017;103:341-7.

19. Li Y, Wu ZG, Li XK, Guo Z, Wu SH, Zhang YQ, et al. A polycaprolactone-tricalcium phosphate composite scaffold as an autograft-free spinal fusion cage in a sheep model. Biomaterials. 2014;35(22):5647-59.

20. Yu Y, Wang J, Liu C, Zhang B, Chen H, Guo H, et al. Evaluation of inherent toxicology and biocompatibility of magnesium phosphate bone cement. Colloids Surf B Biointerfaces. 2010;76(2):496-504. 
21. Witte F, Kaese V, Haferkamp H, Switzer E, Meyer-Lindenberg A, Wirth CJ, et al. In vivo corrosion of four magnesium alloys and the associated bone response. Biomaterials. 2005;26(17):3557-63.

22. Zreiqat $H$, Howlett $C R$, Zannettino A, Evans P, Schulze-Tanzil G, Knabe $C$, et al. Mechanisms of magnesium-stimulated adhesion of osteoblastic cells to commonly used orthopaedic implants. $\mathrm{J}$ Biomed Mater Res. 2002;62(2):175-84.

23. Daentzer D, Floerkemeier T, Bartsch I, Masalha W, Welke B, Hurschler C, et al. Preliminary results in anterior cervical discectomy and fusion with an experimental bioabsorbable cage - clinical and radiological findings in an ovine animal model. Springerplus. 2013;2:418.

24. Toth JM, Wang M, Scifert JL, Cornwall GB, Estes BT, Seim HB 3. Evaluation of 70/30 D,L-PLa for use as a resorbable interbody fusion cage. Orthopedics. 2002;25(10 Suppl):s1131-40. rd, et al.

25. Steffen T, Tsantrizos A, Fruth I, Aebi M. Cages: designs and concepts. Eur Spine J. 2000;9(Suppl 1):89-94.

26. Wei J, Jia J, Wu F, Wei S, Zhou H, Zhang H, et al. Hierarchically microporous/macroporous scaffold of magnesium-calcium phosphate for bone tissue regeneration. Biomaterials. 2010;31(6):1260-9.

\section{Figures}



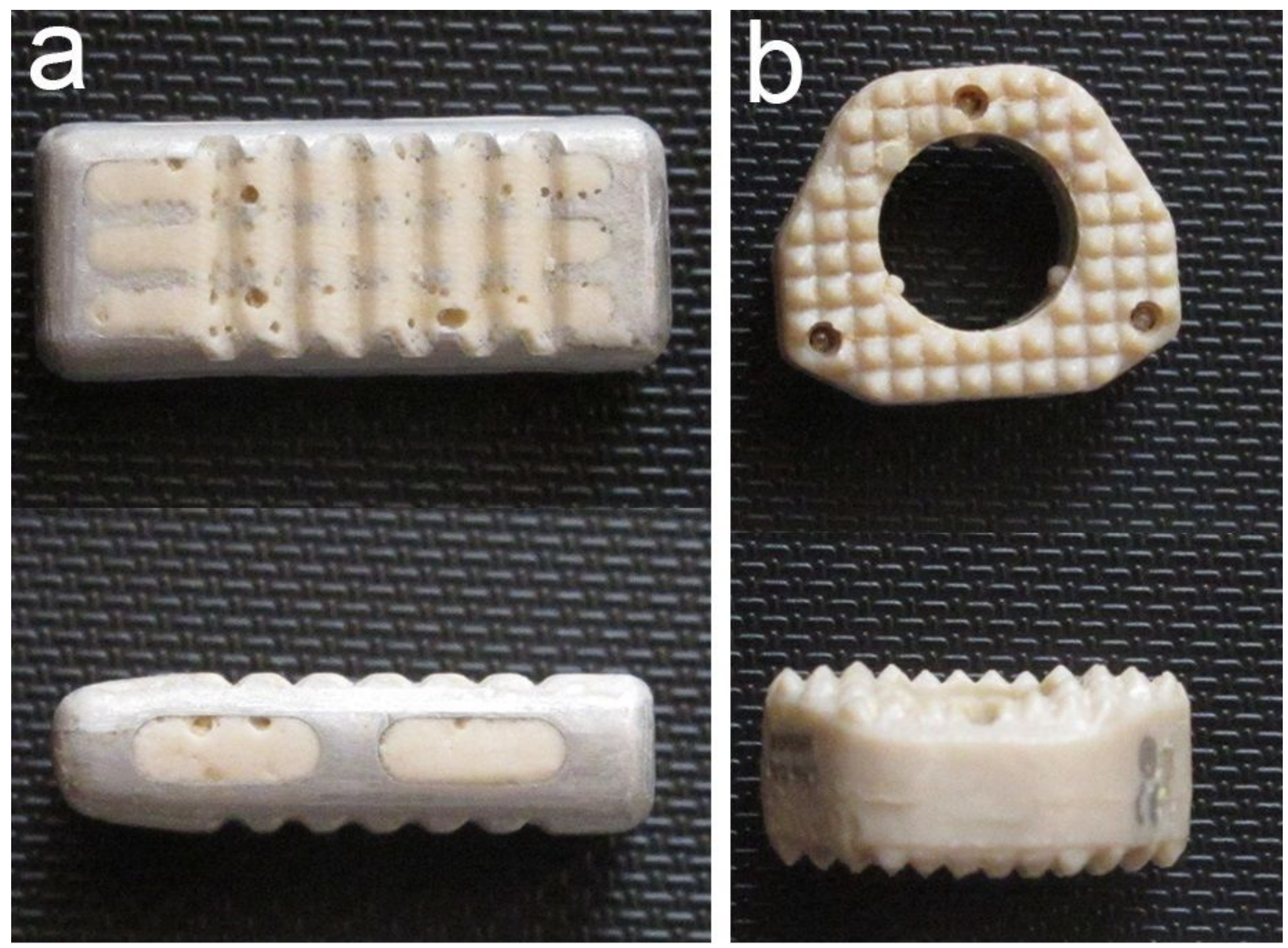

Figure 1

Implants tested in the study.(a), MgロMPC cage; (b), PEEK cage 


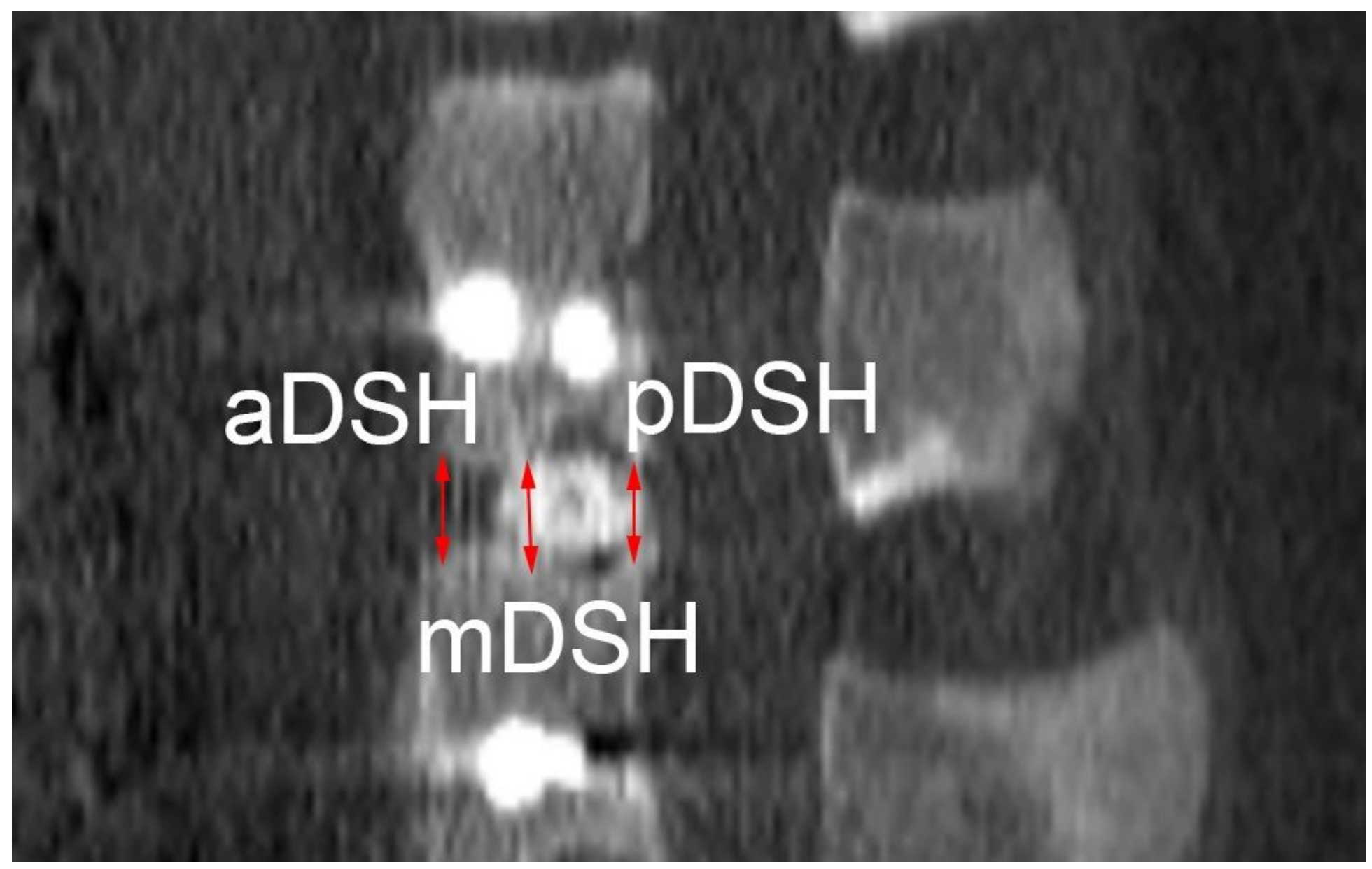

\section{Figure 2}

Measurement of anterior disc space height (aDSH), middle disc space height ( $\mathrm{mDSH}$ ), and posterior disc space height (pDSH) from the middle sagittal CT view of L4/L5 at 6 weeks after the operation 


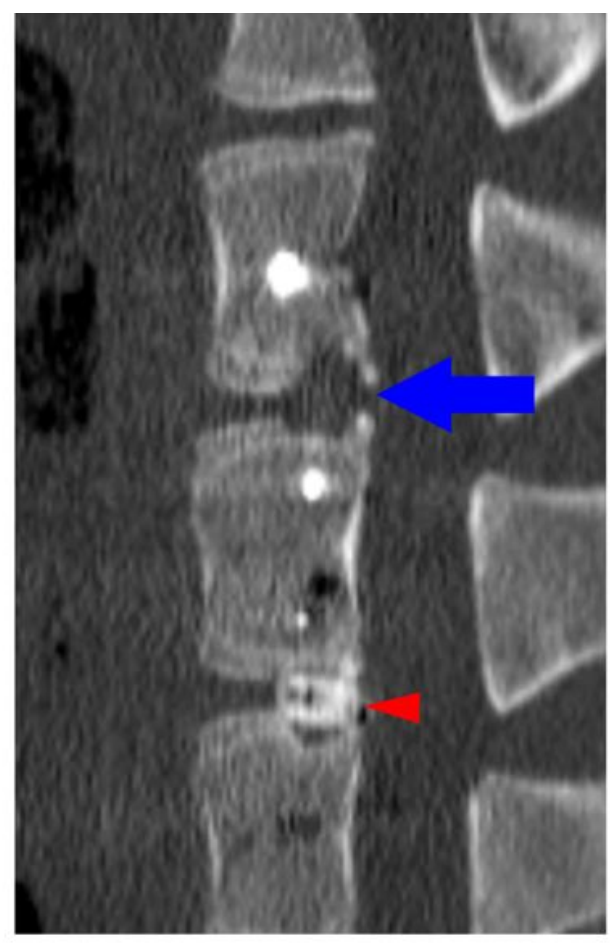

6 weeks

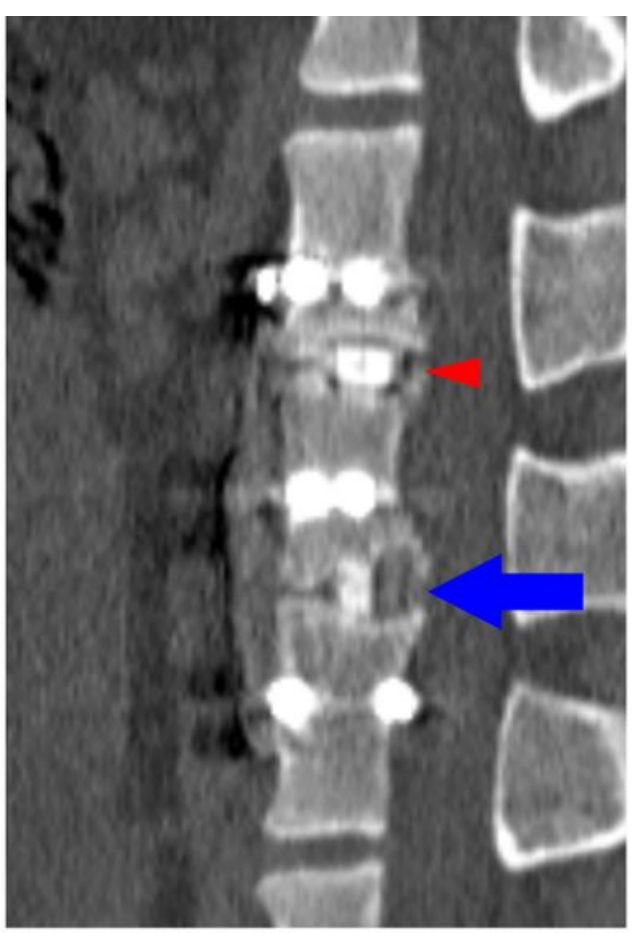

12 weeks

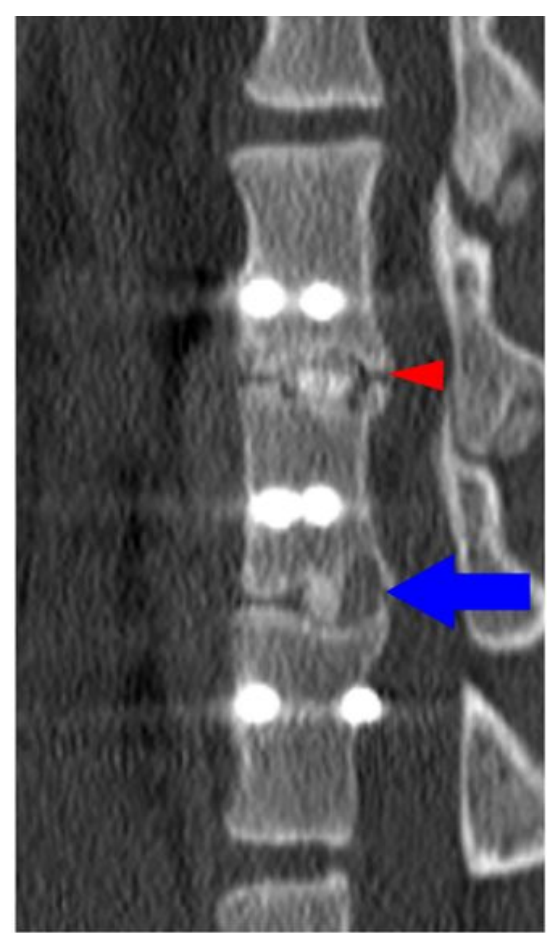

24 weeks

\section{Figure 3}

The middle sagittal CT view of L3/L4 and L4/L5 with implantation of PEEK cage (blue arrow) and MgMPC cage (red triangle) at 6,12 , and 24 weeks after the operation

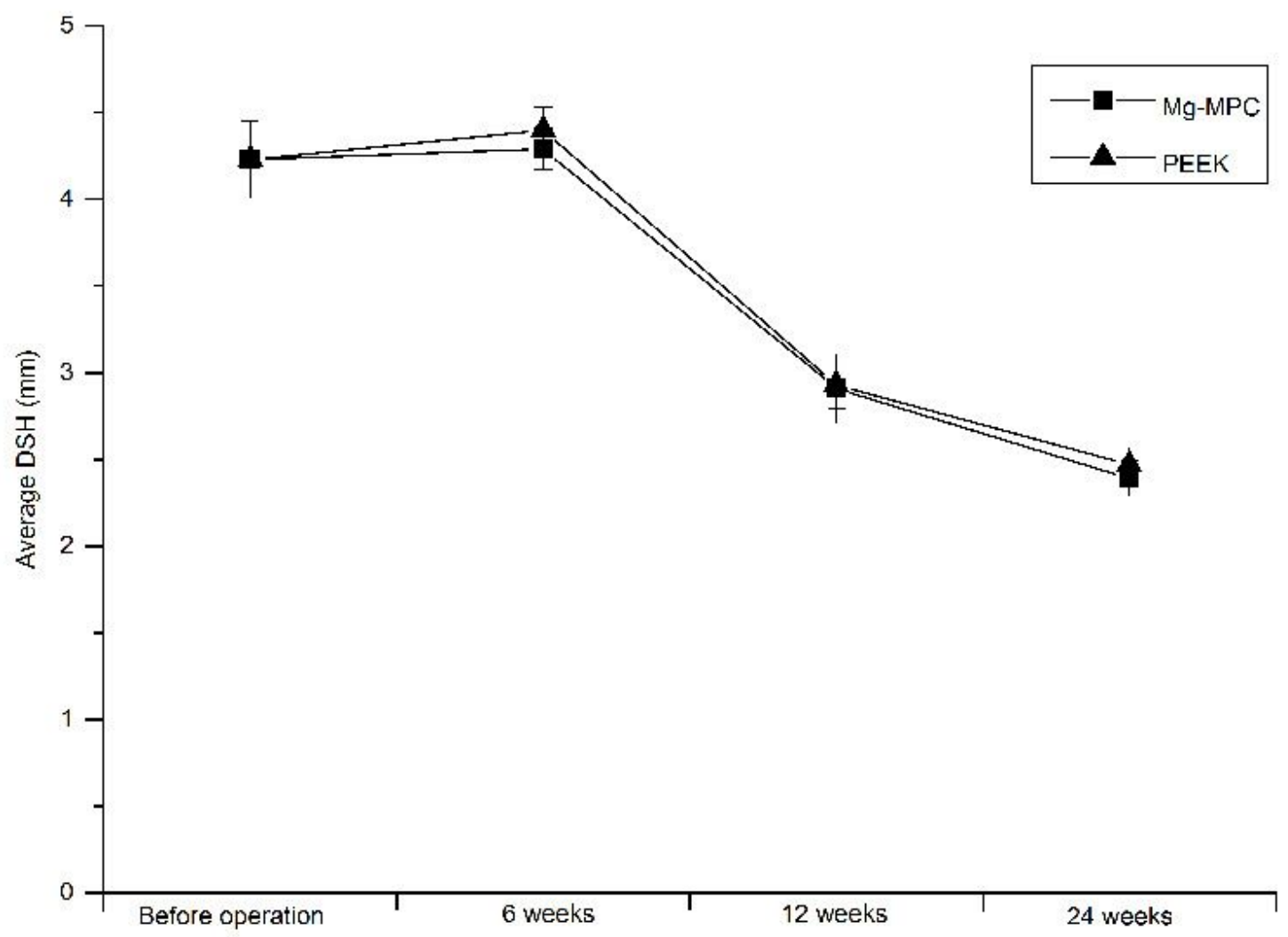


Figure 4

Average DSH on the sagittal CT scan of the two groups throughout the observation period

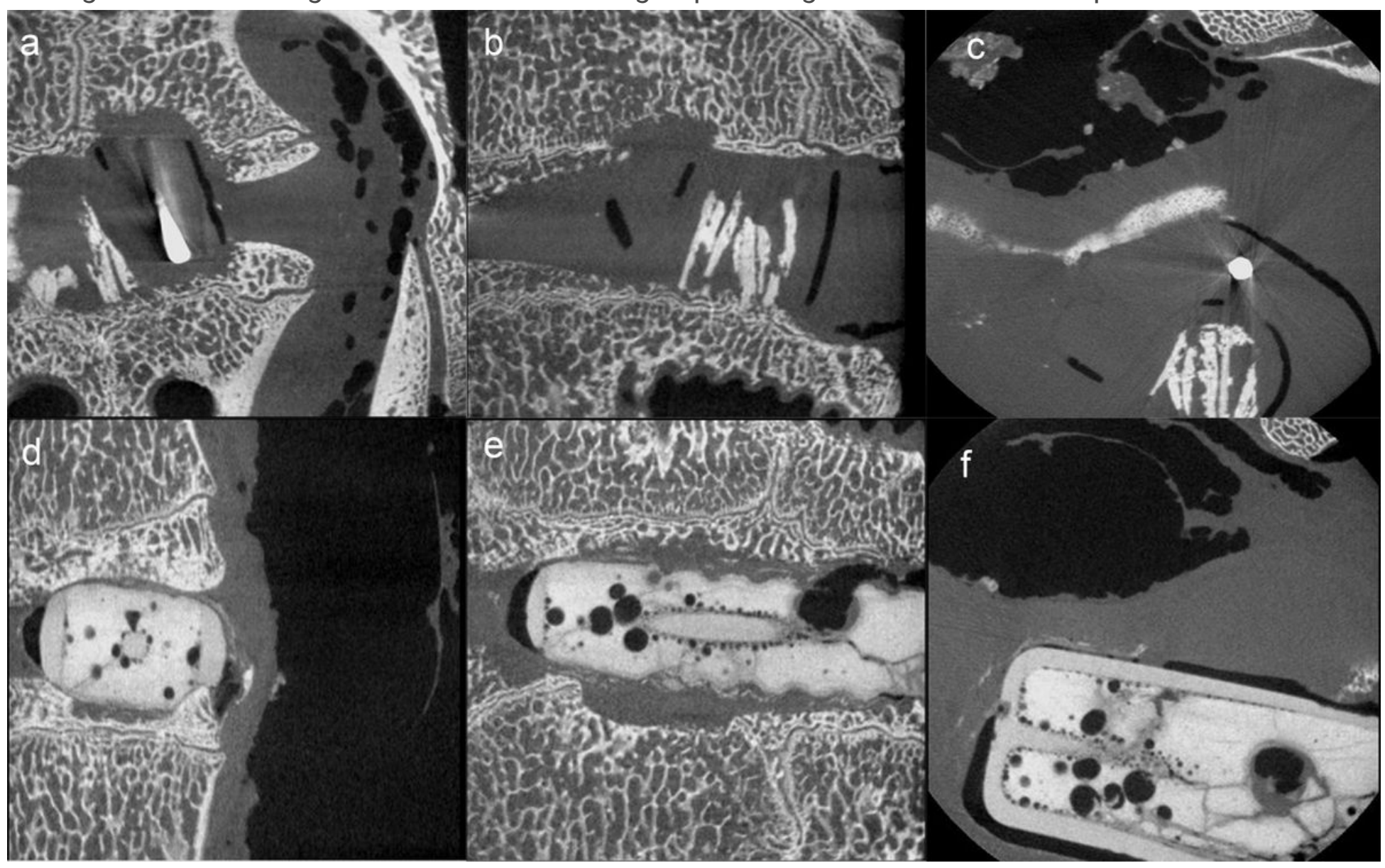

\section{Figure 5}

Micro-CT scans at 6 weeks after the operation in the PEEK group (a: sagittal view, b: coronal view, c: axial view) and the Mg-MPC group (d: sagittal view, e: coronal view, f: axial view). 


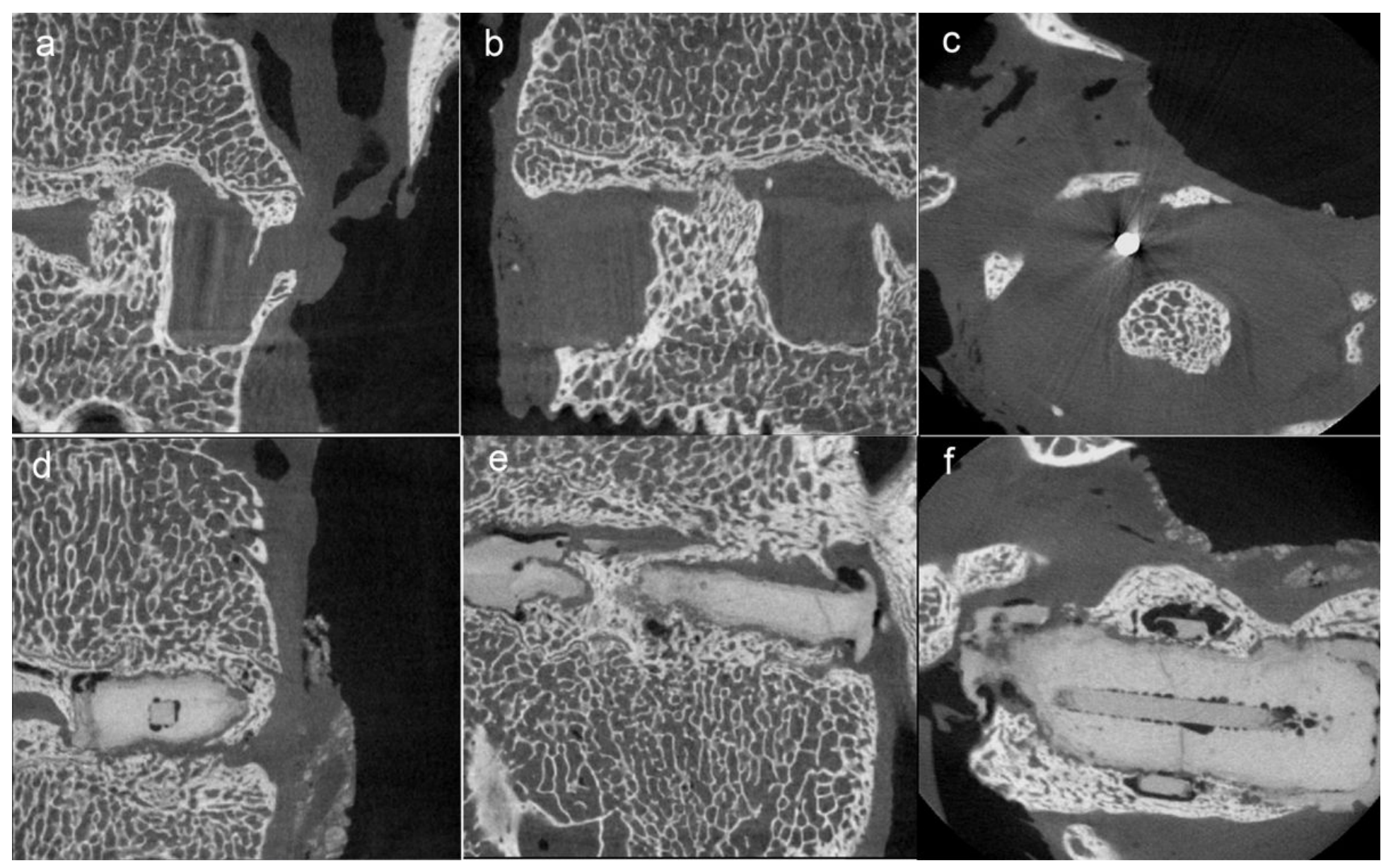

Figure 6

Micro-CT scans at 24 weeks after the operation in the PEEK group (a: sagittal view, b: coronal view, c: axial view) and the Mg-MPC group (d: sagittal view, e: coronal view, f: axial view). 


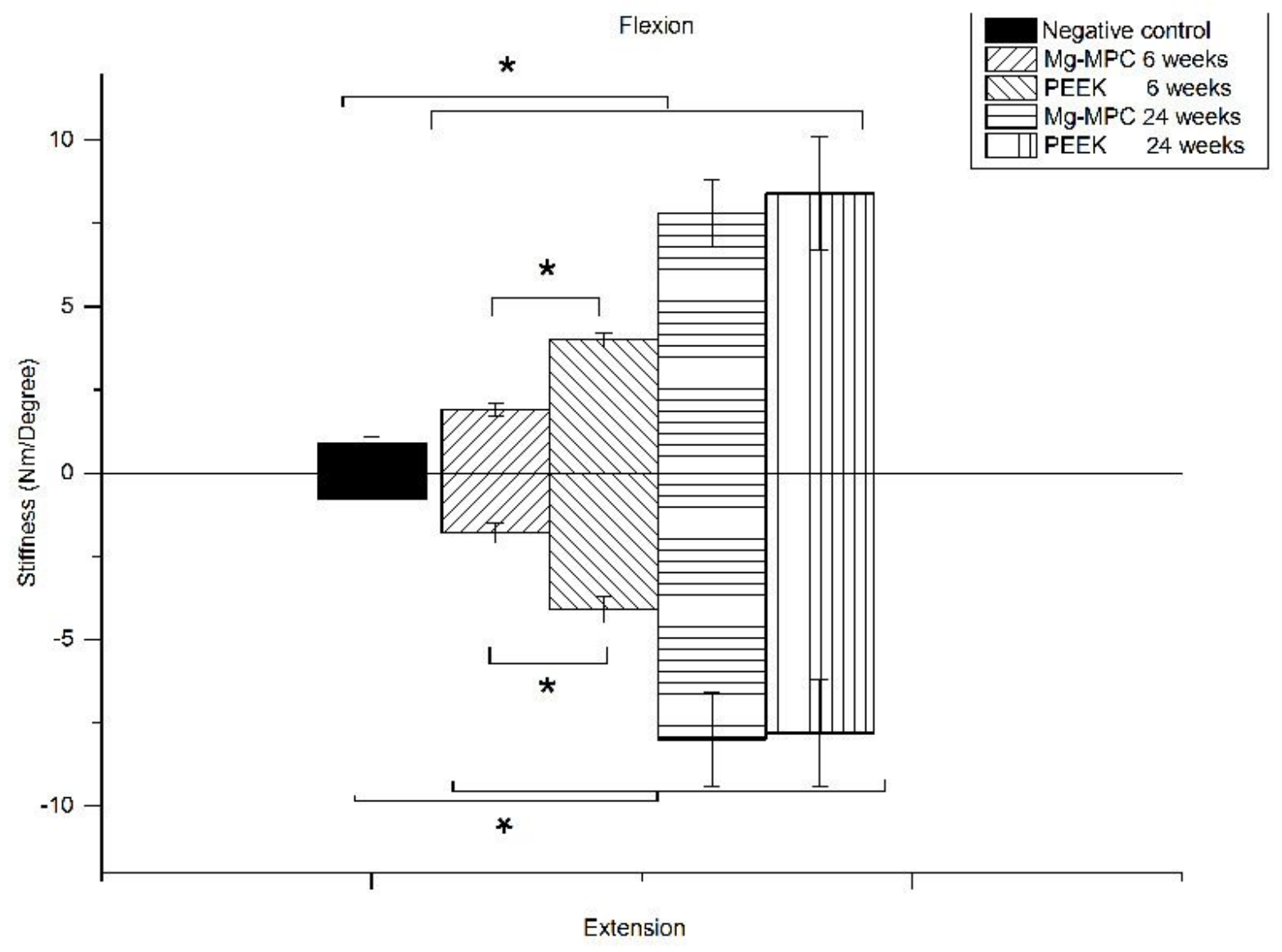

Figure 7

The stiffness analysis in flexion and extension. ${ }^{*} \mathrm{P}<0.05$ 


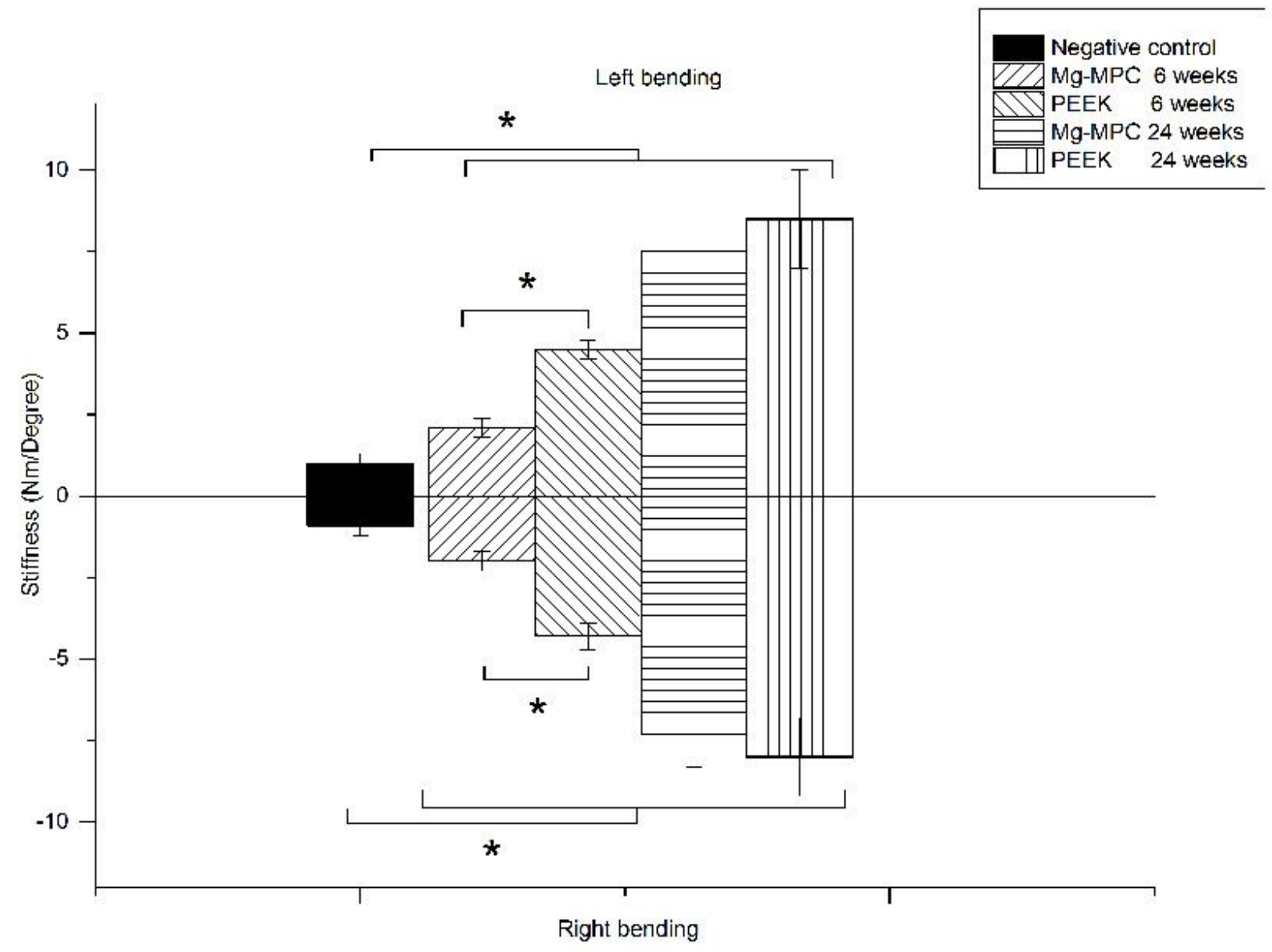

Figure 8

The stiffness analysis in lateral bending. ${ }^{*} P<0.05$ 


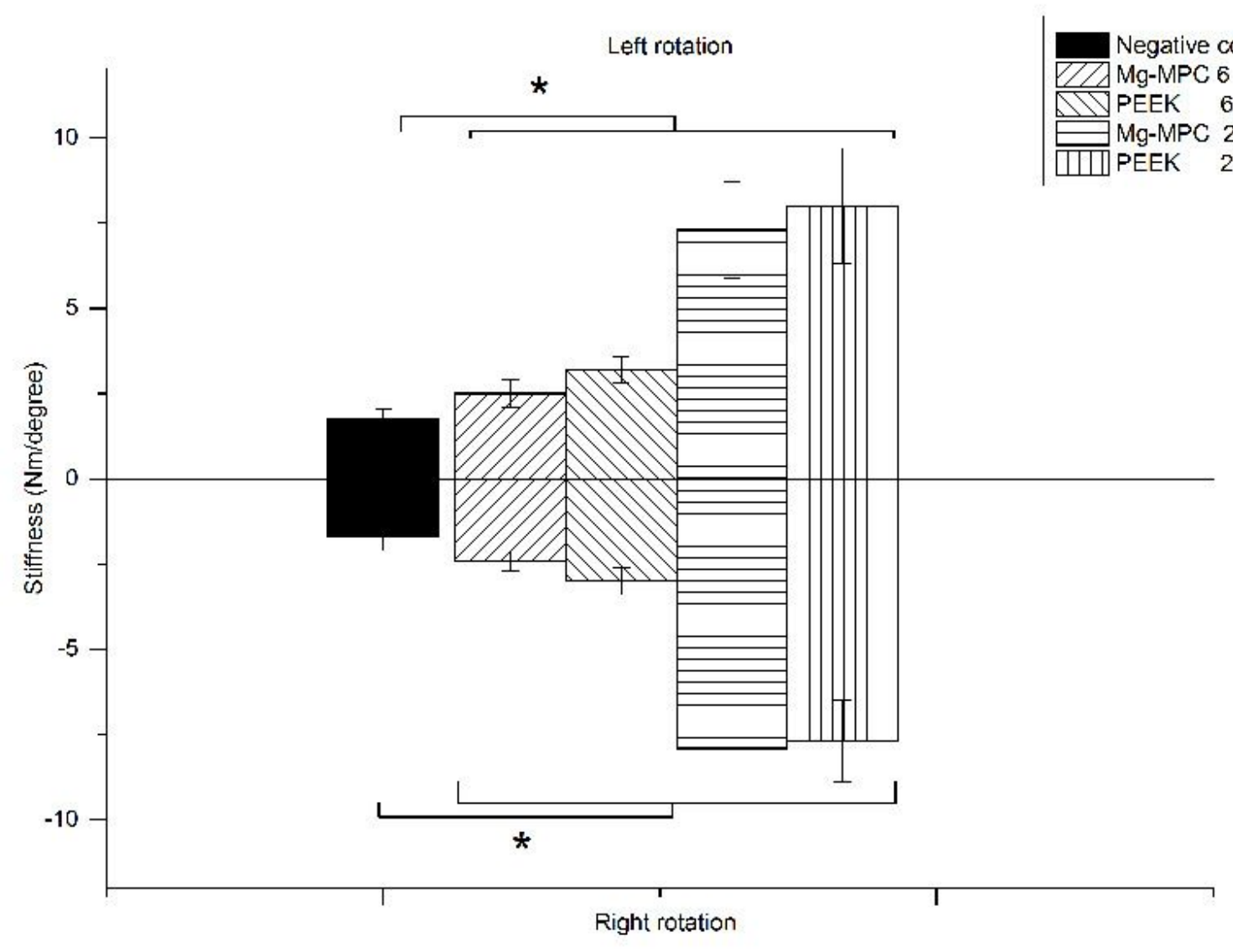

Figure 9

The stiffness analysis in rotation mode. ${ }^{*} \mathrm{P}<0.05$
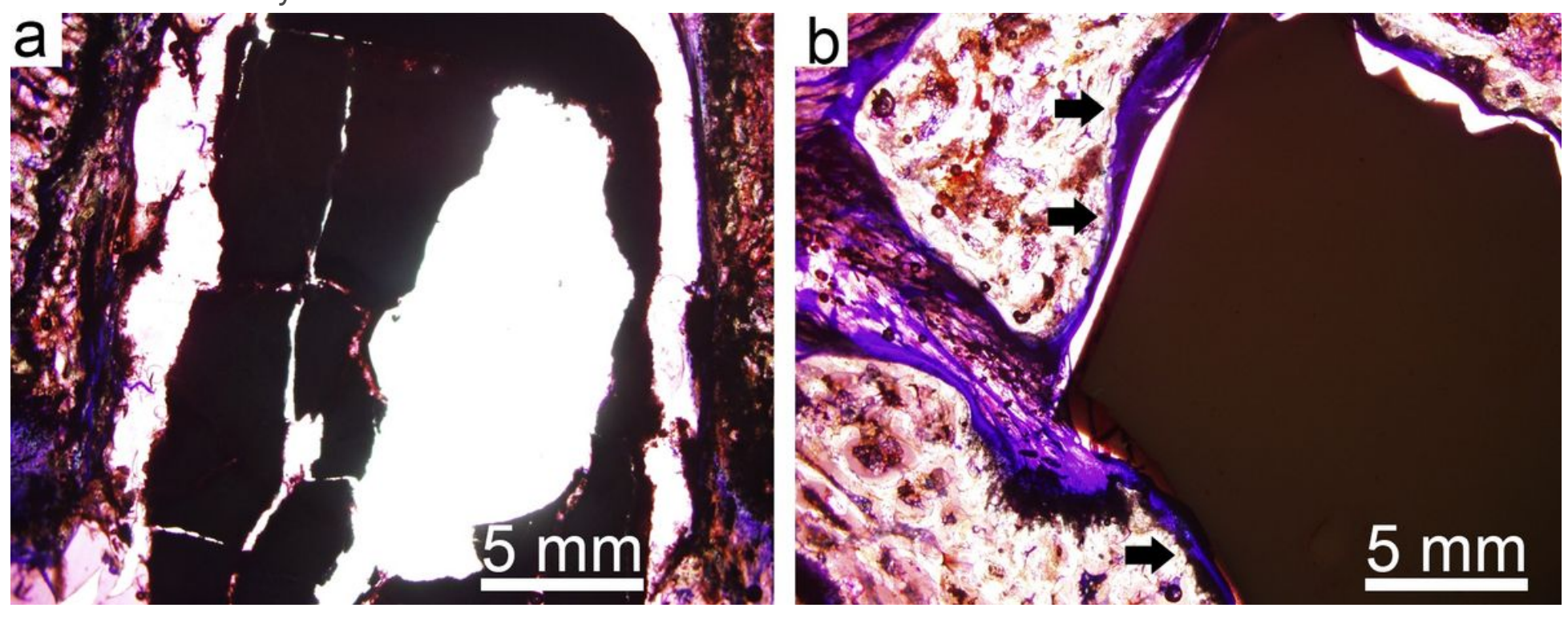

Figure 10

Histological images with Giemsa staining at 6 weeks after the operation (a) in the Mg-MPC group and (b) in the PEEK group. Fibrous tissue (black arrow) formed around the PEEK cage 

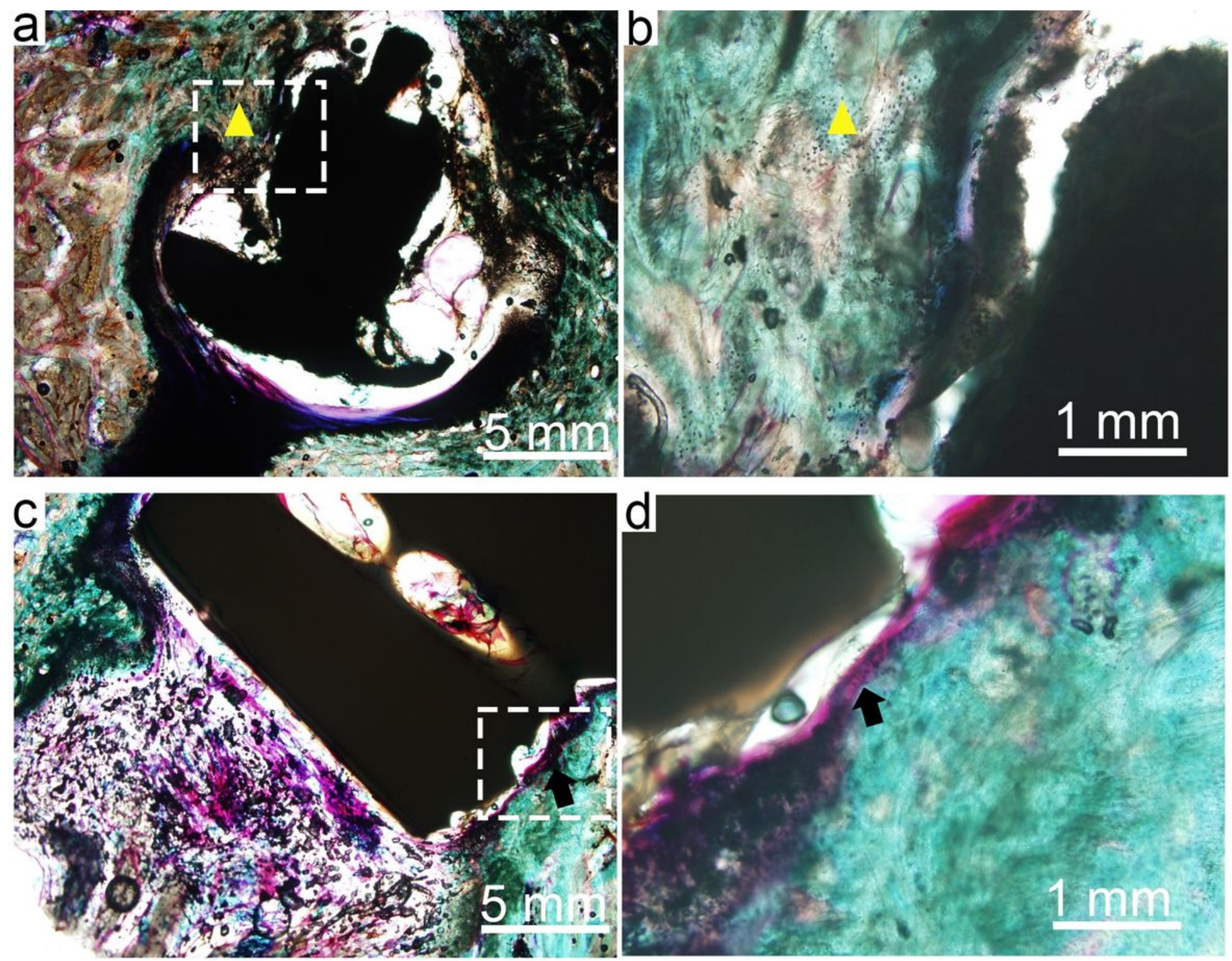

Figure 11

Histological images with Masson trichrome staining at 24 weeks after the operation $(a, b)$ in the Mg-MPC group and $(c, d)$ in the PEEK group. Yellow triangles indicate newly formed bone. Black arrows indicate fibrous tissue 